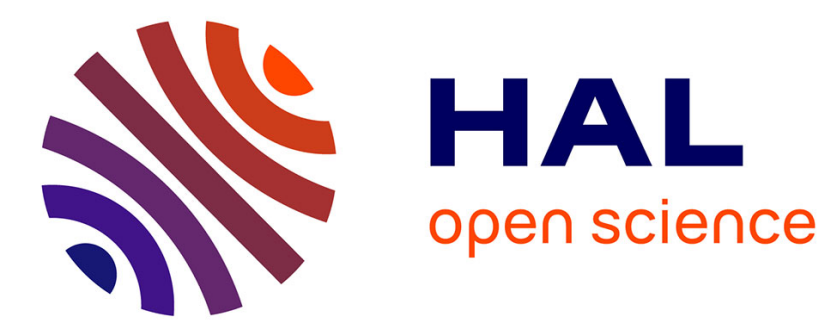

\title{
Galloping instability and control of a rigid pendulum in a flowing soap film
}

\author{
Alessandro Orchini, Hamid Kellay, Andrea Mazzino
}

\section{To cite this version:}

Alessandro Orchini, Hamid Kellay, Andrea Mazzino. Galloping instability and control of a rigid pendulum in a flowing soap film. Journal of Fluids and Structures, 2015, 56, pp.124-133. 10.1016/j.jfluidstructs.2015.04.006 . hal-01186764

\section{HAL Id: hal-01186764 \\ https://hal.science/hal-01186764}

Submitted on 25 Aug 2015

HAL is a multi-disciplinary open access archive for the deposit and dissemination of scientific research documents, whether they are published or not. The documents may come from teaching and research institutions in France or abroad, or from public or private research centers.
L'archive ouverte pluridisciplinaire HAL, est destinée au dépôt et à la diffusion de documents scientifiques de niveau recherche, publiés ou non, émanant des établissements d'enseignement et de recherche français ou étrangers, des laboratoires publics ou privés.

\section{다(1)(2)}

Distributed under a Creative Commons Attribution - ShareAlikel 4.0 International 


\title{
Galloping instability and control of a rigid pendulum in a flowing soap film
}

\author{
Alessandro Orchini ${ }^{\mathrm{a}, *, 1}{ }^{2}$ Hamid Kellay ${ }^{\mathrm{b}}$, Andrea Mazzino ${ }^{\mathrm{c}, \mathrm{d}}$ \\ a Physics Department, University of Genova, Via Dodecaneso 33, 16146 Genova, Italy \\ ${ }^{\mathrm{b}}$ Univ. Bordeaux, Laboratoire Ondes et Matière d'Aquitaine (UMR 5798 CNRS), 351 cours de la Libération, 33405 Talence, France \\ ${ }^{\mathrm{c}}$ DICCA, University of Genova, Via Montallegro 1, 16145 Genova, Italy \\ ' INFN and CINFAI Consortium, Genova Section, Via Dodecaneso 33, 16146 Genova, Italy
}

Keywords:

Fluid-structure interaction

Galloping

Flow control

\begin{abstract}
A B S T R A C T
A pendulum suspended in a fast flowing soap film may show sustained oscillations. The conditions necessary for self excited motion to occur are outlined: a flow velocity above a threshold value along with geometrical constraints. The role of vortex shedding is discussed, and the observed instability is shown to be well described by the galloping instability. Experimental results are supported by numerical simulations. Furthermore, we observe that the instability may be suppressed by attaching a long enough filament to the rear of the pendulum.
\end{abstract}

\section{Introduction}

The problem of fluid structure interaction is a highly practical issue as its implications cover a great variety of phenomena, spanning from how organisms swim and harness the energy of diverse flows to the vibrations of vehicles such as a submarine in turbulent water or an airplane in turbulent air. This interaction may be desirable, as in the case of swimming organisms, or detrimental, since it may generate additional drag or cause excessive vibrations in a variety of practical flows and structures. Different approaches and methods have been applied to the study of this problem: experimental observation of swimming strategies of different organisms; numerical simulations of flapping flags; investigation of vortex induced vibrations on structures of engineering interest, like bridges and airplanes (Spalart, 1998; Williamson and Govardhan, 2004; Shelley et al., 2005; Provansal et al., 2005; Lauga and Powers, 2009; Obligado et al., 2013).

When a flow interacts with rigid or flexible bodies, the resulting dynamics can be unsteady and exhibit an oscillatory behavior. In this situation, the possibility of harvesting energy from the flow becomes possible, e.g., by means of devices that convert mechanical energy into electric current (Allen and Smits, 2001; Boragno et al., 2012; Orchini et al., 2013). Many studies have focused on how a wake affects the shape of deformable structures such as a flexible surface (Allen and Smits, 2001) or a fish body (Liao et al., 2003). In the swimming fish case, an adaptation of the swimming strategy to the presence of vortices has been documented by Liao et al. (2003); even for a passive deformable object some passive propulsion has been observed by Beal et al. (2006). Besides its applicability, the fluid structure interaction poses fundamental problems. For example, recent studies have outlined a symmetry breaking mechanism that, so far, has eluded our intuitive understanding of how flows affect the shape and conformation of flexible objects (Bagheri et al., 2012; Lacis et al., 2014).

\footnotetext{
* Corresponding author.

E-mail address: ao352@cam.ac.uk (A. Orchini).

${ }^{1}$ Current address: Department of Engineering, University of Cambridge, Cambridge CB2 1PZ, United Kingdom.
} 
We investigate the stability of a system formed by coupling a pendulum shaped rigid structure and a flowing soap film. Soap film flows being two dimensional, their use allows us to limit the number of degrees of freedom of the system, and to use common visualization and analysis techniques to investigate both the flow field and the embedded objects dynamics (Kellay et al., 1998; Kellay and Goldburg, 2002; Rutgers et al., 2001; Amarouchene and Kellay, 2005; Zhang et al., 2000). Fast flowing soap films have been widely used to study fluid structure interactions in many different situations, e.g., the stability of flexible filaments both in the laminar (Zhang et al., 2000; Jia and Yin, 2008) and turbulent (Amarouchene and Kellay, 2005) regimes, or flag like instabilities (Zhu and Peskin, 2002, 2003; Argentina and Mahadevan, 2004; Lemaitre et al., 2005). More recent experiments have shown the existence of sustained oscillations of light flexible pendulums due to the vortex shedding from the disk (Bandi et al., 2013). Two main differences characterize our analysis with respect to the one carried out by Bandi et al. (2013): the pendulums considered in this study are rigid and heavy, so that their weight always dominates over added mass effects. Under these conditions, we observe an instability in the quasi two dimensional fluid structure system: for a range of geometrical parameters, and above a well defined threshold velocity, the system is unstable, and, after a transient behavior, shows sustained, large amplitude, low frequency oscillations. Although we have mentioned the possibility to harvest energy from the resulting oscillatory dynamics, in this work we will not discuss how the configuration can be optimized for this goal, but will only discuss the origin of the instability and how it can be controlled.

By means of experimental observations and with the aid of a simple analytical model, we show that this instability can be understood in terms of the galloping mechanism, which is characterized by oscillations in the direction transverse to the flow. Galloping arises depending on the slope of the moment coefficient of the structure versus the angle of attack: the latter property strongly depends on the detailed geometrical shape of the pendulum. Numerical simulations have been performed to validate the analytical model. Additional observations on the effects of a flexible filament attached to the rear of the pendulum are also reported. For short lengths, the instability persists, its amplitude does not change, and the filament and the pendulum oscillate in synchrony. However, when the length of the filament is larger than a threshold value, the galloping instability is inhibited and the rigid system comes to rest, whereas the flexible filament continues to flap.

\section{Experimental setup}

The experiments described in this paper were carried out in a soap film channel into which a rigid pendulum was embedded in the flow direction. To perform the experiments we used a vertical setup (see Fig. 1), as described by Kellay et al. (1995, 1998), Rutgers et al. (2001), and Kellay and Goldburg (2002).
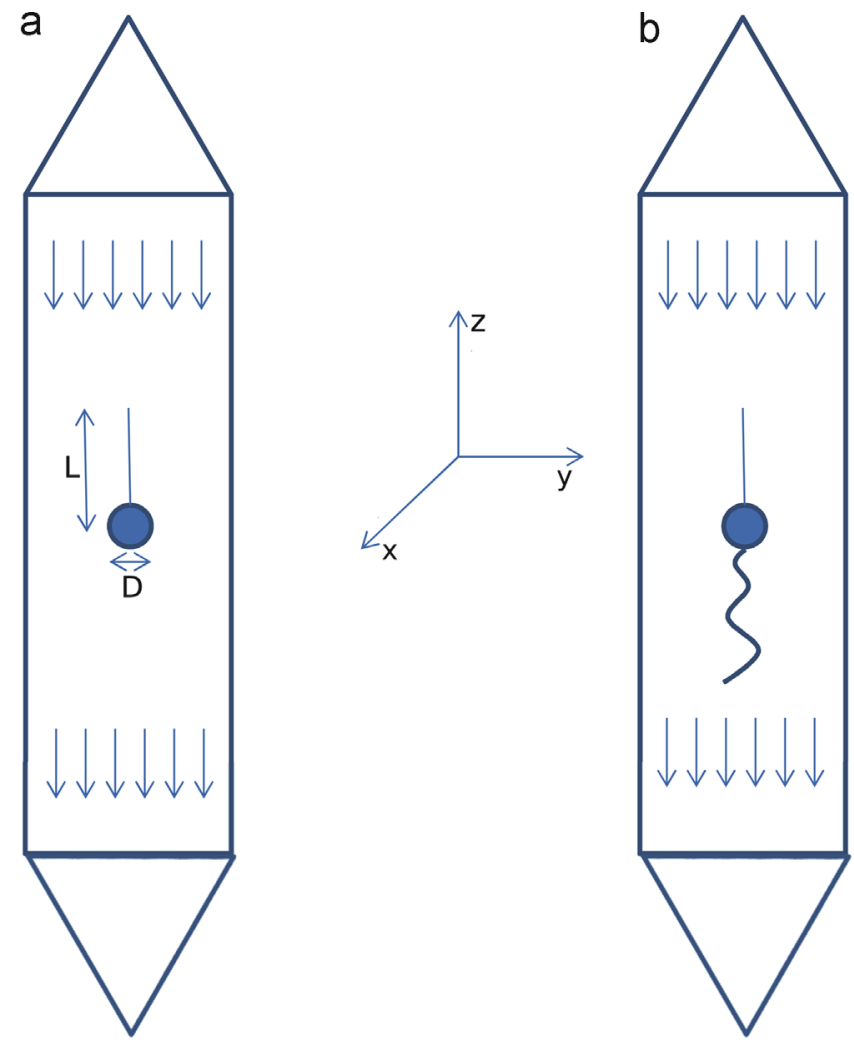

Fig. 1. Schematic of the soap film channel where the water-soap solution flows between two nylon wires. Soap water is injected at the top of the channel and the flow direction is indicated by arrows. (a) An embedded rigid pendulum Sections 3, 4, (b) rigid pendulum with a flexible filament attached Section 5. 
The device dimensions are approximately $20 \times 140 \mathrm{~cm}$ in the $\hat{\mathbf{y}} \hat{\mathbf{z}}$ directions respectively. The width was sufficiently large to minimize the interaction between the pendulum and the sides of the soap film channel. A volumetric $2 \%$ water soap ${ }^{1}$ solution is used to create the soap film flowing between two parallel nylon wires. A pump provides solution recirculation and controls its flow rate, which can be varied between 0.1 and $0.5 \mathrm{ml} / \mathrm{s}$, thus controlling the average velocity in the soap film channel, which ranges between 70 and $240 \mathrm{~cm} / \mathrm{s}$. Velocity was measured by means of a Laser Doppler Velocimeter. By measuring the volumetric flow rate, the mean velocity across the channel, and using mass conservation, we can infer the average thickness $h$ of the soap film, which turns out to be approximately $h=5 \pm 2 \mu \mathrm{m}$ over a wide range of velocities. Near the middle of the film, a thin nylon wire (diameter $\approx 100 \mu \mathrm{m}$ ) extending in the $\hat{\mathbf{x}}$ direction crosses the soap film plane, and is used to hang the pendulum.

The pendulum is composed of a long, thin rod of length $L_{r}$ connected to a thin disk of radius $R$. The mass of the disk and its radius, as well as the mass, length and diameter of the rod, can be varied. Hollow disks were used to modify the mass of the pendulum without modifying its diameter. We also used flexible filaments (thin threads of diameter $200 \mu \mathrm{m}$ made of cotton or nylon) of various lengths, attached to the rear of the disks as shown in the schematic on the right in Fig. 1.

Pendulum and flow dynamics is followed by means of video imaging. The collected videos were post processed to determine the frequency and amplitude of the oscillations, as well as to study the role of vortex shedding. Movies at a few hundred frames per second are sufficient to properly sample the flow structure and rigid body dynamics.

\section{Observations}

In many configurations the pendulum is static and linearly stable, showing no self induced oscillations despite the fact that vortices are shed from the disk. In these configurations, if the pendulum is perturbed from its rest position, the amplitude of the induced oscillation decays in time and the pendulum returns to its static, stable configuration, showing that subcritical phenomena are not found for this system.

However, under some conditions the system becomes linearly unstable and after a transient the pendulum exhibits self excited, sustained limit cycle oscillations. During an oscillation cycle, the flow pattern around the pendulum is quite complex with vortices being shed by the disk but also from the top of the pendulum rod as shown in Fig. 2 . The figure shows the formation and roll down of vortices formed at the top of the pendulum during a quarter of the oscillation period ( $T / 4)$ as the oscillation angle of the pendulum varies between $\theta=\left[0, \theta_{\max }\right]$. As the pendulum swings upwards and beyond a certain angle (roughly $\theta_{\max } / 2$ ), vortices are shed from the rod leading edge. These vortices are then advected downstream by the soap flow and reach the disk after a characteristic transit time, which is roughly given by $\tau \approx L / U$, where $L=L_{r}+R$. Whether these vortices enhance the oscillations or induce their damping depends on how and when they interact with the disk. Although one needs to properly characterize the interplay between the vortex shedding and the dynamically induced moments on the disk in order to quantitatively understand the connection between the transit time and the instability's onset, one may anyway expect a link between the characteristic quantities $\tau, L, U$ and the onset of the oscillations.

Indeed, we find that an onset condition for the instability is that the velocity of the flow has to be above a threshold value $U_{t h}$. A representative case of the threshold velocity dependence on the geometrical parameters is shown in Fig. 3: $U_{t h}$ increases with the characteristic length of the pendulum and can be approximated by the power law $U_{t h} \propto L^{1 / 2}$. In Fig. 3 we also plot the oscillation frequency of the pendulum $f$ at the threshold velocity: it decreases with the pendulum characteristic length approximately as $L^{-1 / 2}$. We observe that the period of oscillations $T$ is always consistent with the compound pendulum period, which for small oscillation angles is given by $T=2 \pi \sqrt{ } I / M g b$, where $I$ is the moment of inertia of the pendulum with respect to the anchoring point, $b$ is the distance between the pivot point and the center of mass, and $M$ is the total mass of the pendulum. In most cases, the rigid body mass is concentrated in the disk, and the expression for the period simplifies to $T \approx 2 \pi \sqrt{ } L / g$. In dynamical terms, this means that inertial effects are determining the frequency of oscillations. Nevertheless, the presence of the fluid is necessary for the instability to arise.

Using the power law expressions we just discussed, we also find empirically that the characteristic transit time at the threshold velocity is $\tau_{\text {th }}=L / U_{\text {th }} \approx T / 20$, which gives

$$
U_{t h} \approx \frac{10}{\pi} \sqrt{ } g L
$$

However, it remains a challenging task to quantitatively connect this value with the vortex shedding dynamics.

We now describe what happens to the oscillations of the pendulum as the velocity $U$ approaches $U_{t h}$. At velocities lower than $U_{t h}$, if the pendulum is perturbed from the vertical position it starts to oscillate, but the amplitude of the oscillation decays with a well defined decay rate, and the pendulum returns back to the equilibrium position. This is shown in the upper left inset of Fig. 4: the oscillation amplitude decays exponentially in time, giving a well defined decay rate for the damping of the pendulum. As the velocity approaches $U_{t h}$, the transient response of the pendulum to disturbances changes. Instead of a single decay, it shows a two phase decay; both phases are exponential decays, as shown in the upper right inset of Fig. 4 . The late time decay is faster than the initial one. Also, the late time decay rate does not vary while increasing the mean velocity, and it is always found to have a value of about $0.1 \mathrm{~s}^{1}$ for the example shown here. This is consistent with the viscous damping of a disk of mass $m$, thickness $h_{\text {disk }}=2 \mathrm{~mm}$ in a thin film of viscosity $\eta$ close to that of water $\left(4 \pi \eta h_{\text {disk }} / 2 \mathrm{~m}\right)$ : the late time decay is

\footnotetext{
${ }^{1}$ Liquid detergent Fairy Dreft from Procter and Gamble.
} 


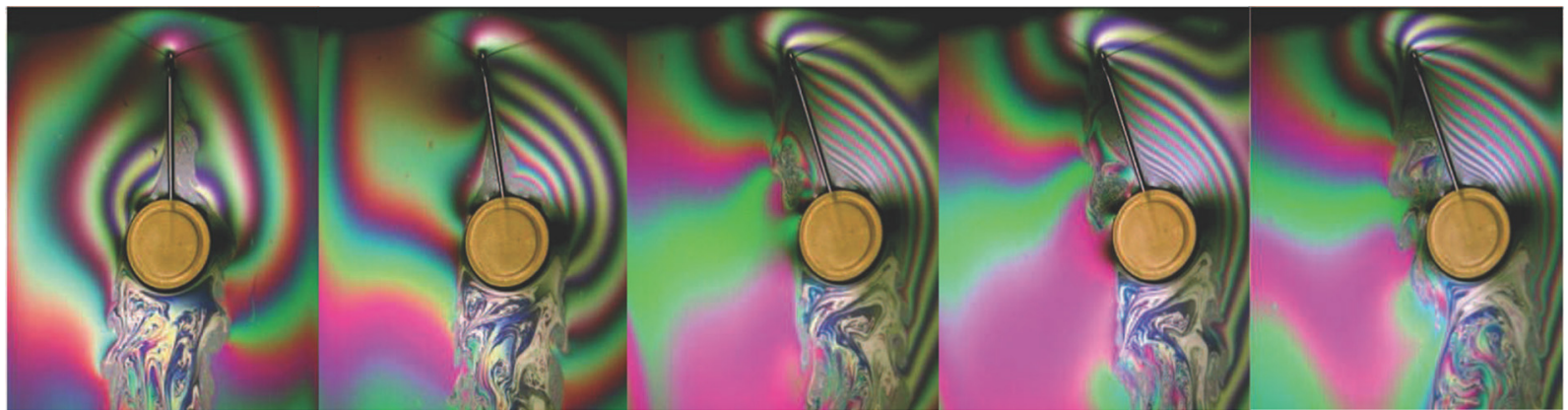

Fig. 2. Sequence of images over a quarter of a self-induced limit cycle oscillation showing vortex shedding from the leading edge. Images are taken at $t \quad 0,21,50,54,82 \mathrm{~ms}$; period $T 330 \mathrm{~ms}$. A plastic disk of diameter $1 \mathrm{~cm}$, thickness $1 \mathrm{~mm}$, and a stainless steel rod of length $1.7 \mathrm{~cm}$ is used. Colors reflect variations in the local soap film thickness (Chomaz, 2001; Amarouchene and Kellay, 2004). (For interpretation of the references to color in this figure caption, the reader is referred to the web version of this paper.)

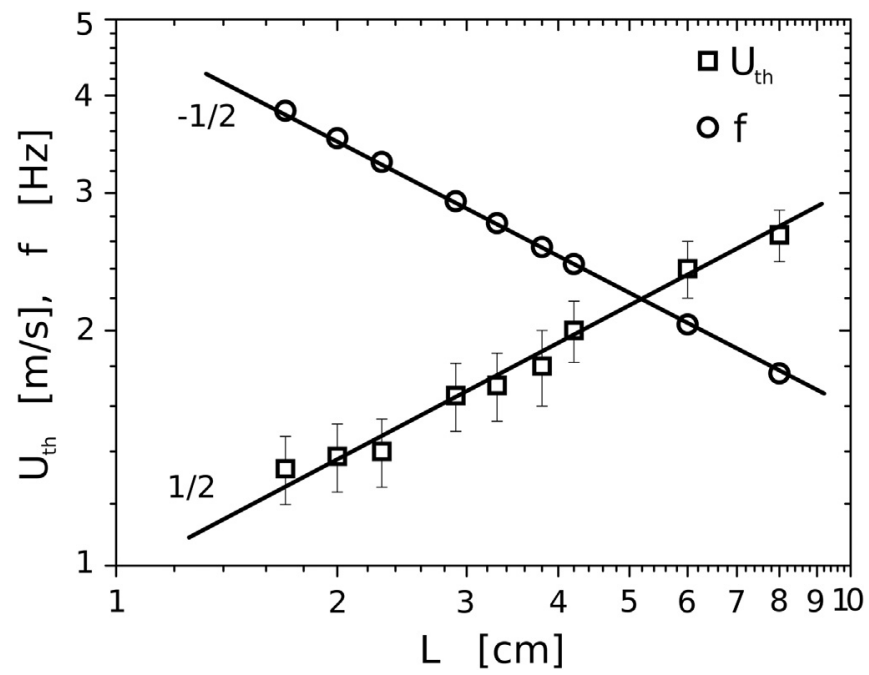

Fig. 3. Threshold velocity and oscillation frequency for a hollow steel disk of thickness $0.8 \mathrm{~mm}$ with $D \quad 2 R \quad 0.6 \mathrm{~cm}$ and $m \quad 0.11 \mathrm{~g}$. The rod was made of a thin stainless steel cylinder (and $D_{r} \approx 0.5 \mathrm{~mm}$ ) whose length $L_{r}$ was varied. The lower boundary of the error bars corresponds to the highest velocity for which the system was stable, and the upper boundary to the smallest velocity for which the system was unstable.

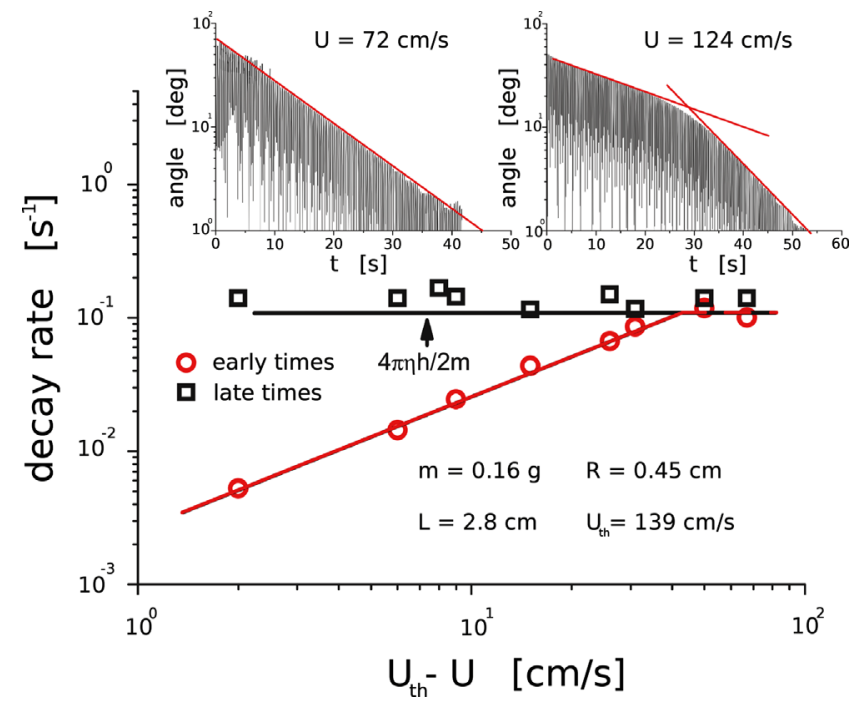

Fig. 4. Decay rates of oscillation in damped cases. Upper insets show typical time series behavior of the angular amplitude at (left) low velocity and (right) velocity close to the threshold value. The disk is a plastic ring of thickness $2 \mathrm{~mm}$. 


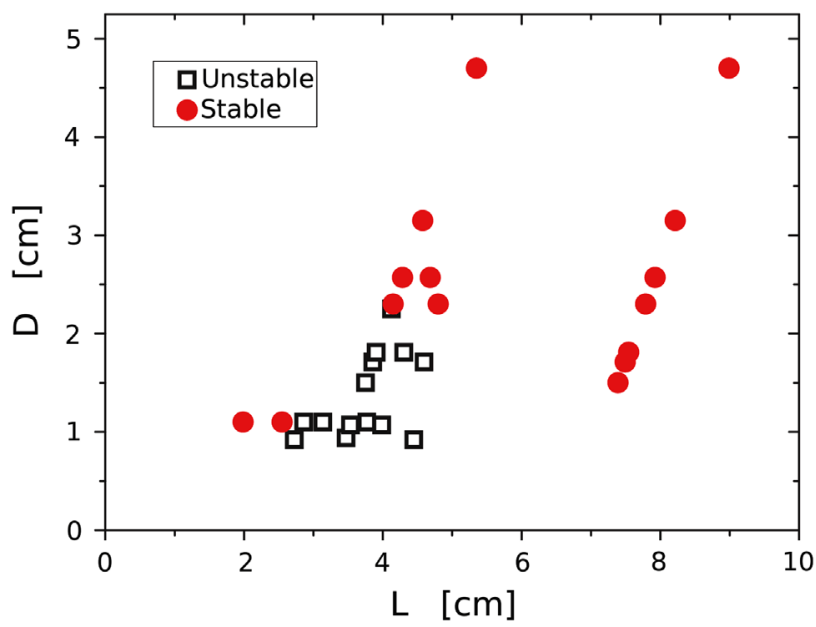

Fig. 5. Stability diagram in the parameter space $L \times D$. Unstable cases are found to lie roughly between the regions $L<50 \mathrm{~mm}$ (threshold velocity condition) and $L>2 D$ (geometrical conditions).

therefore controlled by viscous dissipation. The early time decay rate, however, is dependent on the distance to $U_{t h}$ and goes to zero as $U_{t h}$ is approached from below as ( $U_{t h} U$ ). This suggests that the system's damping coefficient changes sign across the threshold velocity. This can be described with a classical harmonic oscillator model, as we demonstrate in Section 4.

Besides the existence of a threshold velocity, geometrical parameters also play a role in the system's stability. For a fixed velocity $U=180 \mathrm{~cm} / \mathrm{s}$, we show in Fig. 5 a stability diagram in the parameter space $L \times D$. For long rods, the velocity is below the threshold $U_{t h}$ and the system is stable ( $L>0.5 \mathrm{~cm}$ in Fig. 4). Furthermore, self sustained oscillations are observed only if the length $L$ is greater than about twice the disk diameter. This can be interpreted with the following argument: if the disk is too large, the vortex patches shed by the rod's tip fall on top of the disk $(D>L / 2$ in Fig. 4). As a consequence, they do not contribute to lift generation and the system remains stable; on the other hand, if the radius of the cylinder is small, the vortex patches will reach a side of the disk as the pendulum swing upwards, generating lift forces and affecting the system's stability.

\section{Galloping model and numerical validation}

The basic mechanism for sustained oscillations of the rigid pendulum, with no filament attached, can be understood in terms of a quasi steady argument. Indeed, the pendulum dynamics can be described in terms of the second cardinal equation of the dynamics of a solid object subject to the sole rotation degree of freedom:

$$
\theta+2 \zeta \omega_{0} \dot{\theta}+\omega_{0}^{2} \theta=\frac{M_{F}}{I},
$$

where $\theta$ is the oscillation angle, $\omega_{0}=\sqrt{ } g / L$ is the gravitational pendulum angular frequency, $\zeta$ is the damping ratio, $I$ is the moment of inertia with respect to the pivot point, and $M_{F}$ is the fluid induced torque evaluated at the anchoring point.

The fluid torque can be expressed in terms of the nondimensional moment coefficient parameter $C_{M}$ as

$$
M_{F}(\theta, \dot{\theta})=\frac{1}{2} \rho U^{2} D^{2} C_{M}(\theta, \dot{\theta}),
$$

and in general depends on the instantaneous oscillation angle $\theta$ and angular velocity $\dot{\theta}$. Focusing on small amplitude perturbations, we can use a quasi steady argument and define at each instant an effective angle of attack $\alpha$, which takes into account the angular velocity contribution as

$$
\alpha=\theta+\frac{L}{U} \dot{\theta}
$$

where, as customary in the torsional galloping instability analysis (Blevins, 2001), a reference point on the cross section at a characteristic radius L and an angle $\theta$ with respect to the pivot are chosen for evaluation of the angle of attack induced by $\dot{\theta}$.

We then assume that the moment coefficient is a function of the effective angle of attack only. Expanding the expression (3), the first order in $\alpha$ reads

$$
M_{F}=\left.\frac{1}{2} \rho U^{2} D^{2} \frac{d C_{M}}{d \alpha}\right|_{\alpha} \quad 0^{\alpha+\mathcal{O}\left(\alpha^{3}\right)}
$$

The order zero term vanishes because of the symmetry of the structure we are considering. 
Substituting Eqs. (5) and (4) into Eq. (2), and recalling that $\omega_{0}=\sqrt{ } g / L$ and $I \approx M_{D} L^{2}=\pi \rho_{D} D^{2} / 4 L^{2}$, we obtain

$$
\left.\theta+2\left(\zeta \sqrt{\frac{g}{L}} \frac{1}{\pi} \frac{\rho}{\rho_{D}} \frac{U}{L} a\right) \dot{\theta}+\frac{g}{L} \frac{2 \rho}{\pi \rho_{D}} \frac{U^{2}}{L^{2}} a\right) \theta=0
$$

where we have defined $a=\left.\left(d C_{M} / d \alpha\right)\right|_{\alpha} \quad 0$. Note that the $\dot{\theta}$ coefficient now includes both the structural damping term $\zeta \sqrt{ } g / L$ and the fluid induced damping term $\rho U a /\left(\pi \rho_{D} L\right)$ : if the slope of the moment coefficient $a$ is positive, the overall damping coefficient can turn negative, resulting in an instability. This derivation follows the classical instability analysis for torsional galloping of bluff bodies (Blevins, 2001). The emergence of this instability depends on the particular shape of the object and is related to its induced wake arrangement, which must generate a positive torque for positive, small angles of attack.

The onset of the instability is found when the coefficient in front of $\dot{\theta}$ vanishes, assuming that the $\theta$ coefficient remains positive, so that the pendulum behaves as an undamped harmonic oscillator. Under these conditions, one can find an expression for the threshold velocity, which is given by

$$
U_{t h}=\left.\pi \zeta \frac{\rho_{D}}{\rho} \sqrt{ } g L \frac{d C_{M}}{d \alpha}\right|_{\alpha} \quad 0
$$

Note that $U_{\text {th }}$ increases with the pendulum characteristic length as $L^{1 / 2}$, as was qualitatively observed in our experiments (see Fig. 2), and might be compared with the empirical expression (1).

For the threshold velocity to be positive, i.e., for the instability to occur, the slope of the moment coefficient has to be positive at small angles of attack. It is a quite difficult task to determine the sign of $a$ experimentally, as the measurement of the forces acting on the disk is not trivial. We therefore resort to numerical simulations, through which fluid forces on rigid, static bodies $(\alpha=\theta)$ can be conveniently integrated.

The framework of Overture ${ }^{2}$ is used to simulate the flow around a $2 \mathrm{~d}$, static pendulum fixed at various angles of attack in a channel with dimensions similar to the experimental one. Pendulum's static condition is justified by the quasi steady nature of the galloping instability that we are investigating. The main domain is a $9 \times 75 \mathrm{~cm}$ rectangular grid, in which a set of non uniform, overlapping grids is considered to discretize the geometry (see Fig. 6). The resolution of each grid component varies in space: it ranges from a minimum of about $20 \mu \mathrm{m}$ close to the pendulum's surface, where fluid structure interaction is important and forces on the pendulum are integrated, to a maximum of about $2 \mathrm{~mm}$ in the quiescent region far upstream of the pendulum. A total number of about $1.5 \cdot 10^{5}$ grid points were considered. The time step was implicitly set to a value of about $4 \mu$ s.

The pendulum is placed in the middle of the domain, and is constructed by merging a thin plate and a circle grids; no slip boundary conditions are set on its contour, as well as on the left and right sides of the domain. The pendulum's rod length and width are $L_{r}=2.28 \mathrm{~cm}$ and $D_{r}=0.056 \mathrm{~cm}$ respectively, and the circle radius is $R=0.9 \mathrm{~cm}$. These values were chosen to match experimental dimensions. The inlet flow (top of the domain) has no tangential component and constant pressure. An outflow boundary condition is specified on the bottom side of the domain. Following Zhu and Peskin (2002), we add a forcing term to the Navier Stokes equations to account for air resistance effects, which is given by $\lambda \boldsymbol{u}$, where $\lambda$ is the air resistance coefficient, assumed to be constant.

Considering as a computational domain the channel without any embedded rigid body, the steady Navier Stokes equations reduce to

$$
\begin{cases}\nu \frac{d^{2} V}{d y^{2}} \quad \frac{\lambda}{\rho} V \quad g=0 \\ V(y= & d / 2)=V(y=+d / 2)=0,\end{cases}
$$

where $\rho$ is the two dimensional flow density, $g$ the gravitational acceleration, and $d$ the channel width. The solution of Eq. (8) reads

$$
V(y)=V_{0}\left[\begin{array}{ll}
\frac{\cosh \sqrt{\frac{\lambda}{\nu \rho} y}}{\cosh \sqrt{\frac{\lambda}{\nu \rho} \frac{d}{2}}} & 1
\end{array}\right]
$$

where the flow terminal velocity $V_{0}$ is related to the air resistance coefficient via $V_{0}=\rho g / \lambda$. Note that in the simulations the system is perfectly 2 d, i.e., it has zero thickness. The air resistance coefficient is chosen so that the terminal velocity is $V_{0}=1 \mathrm{~m} / \mathrm{s}$, a value representative of the velocities we have investigated in the quasi $2 \mathrm{~d}$ experiments. Solving the Navier Stokes equations (with the air resistance term) in the pressure Poisson formulation using Overture framework, we recover the flow profile (9) in the absence (or far upstream) of the pendulum, thus benchmarking the code. More information on the numerical scheme can be found in Chesshire and Henshaw (1990) and Henshaw (1994); details on our implementation have been presented in Orchini et al. (2013).

\footnotetext{
${ }^{2}\langle$ www.overtureframework.org $\rangle$
} 


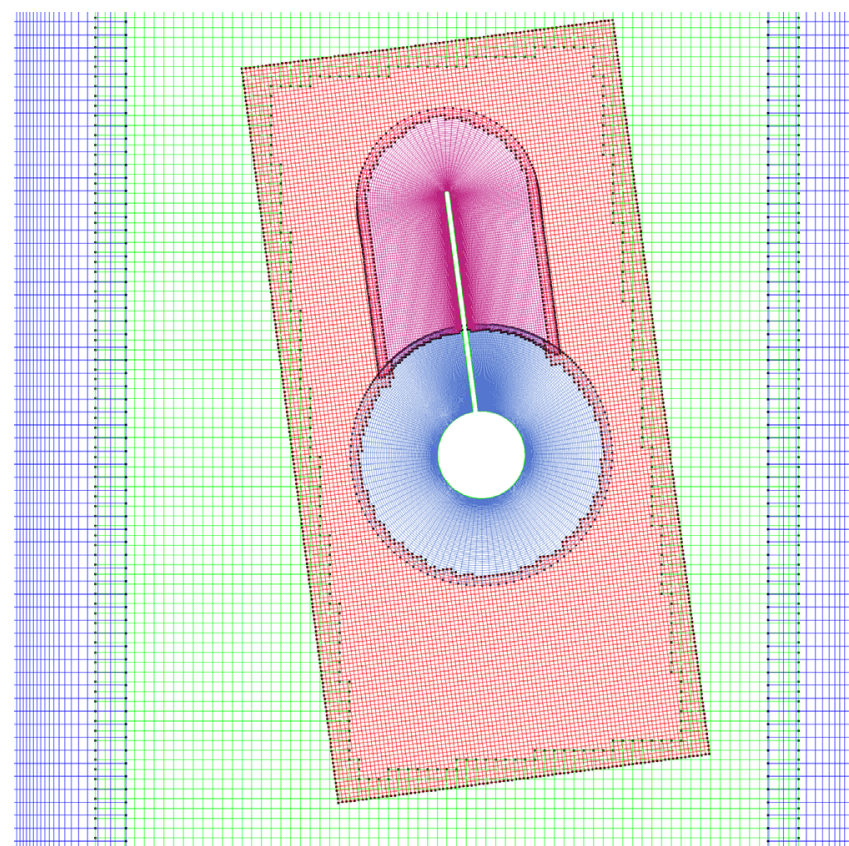

Fig. 6. Detail on the set of overlapping grids generated with Ogen (Henshaw, 1998) and used to discretize the physical domain. The inlet, outlet and side of the channel are not shown in this inset. The governing PDEs are solved on each grid, and the numerical solution is interpolated in the overlapping areas (delimited by small squares). The grids resolution varies spatially, increasing towards the pendulum's physical boundary, on which the fluid-induced torque is integrated.

Although the pendulum's position is fixed, its bluff body nature generates vortex shedding and the resulting flow is unsteady. Therefore, we evaluate fluid forces on the pendulum's surface over many shedding cycles, and we then consider the mean value of the moment coefficient time series as a characteristic value for $C_{M}$.

Fig. 7 shows the behavior of the pendulum's $C_{M}$ evaluated with respect to the anchoring point for different angles of attack; the inset shows the moment coefficient slope at small angles of attack. Its sign is positive, and it is thus consistent with a galloping instability.

We have also simulated the case of the sole rigid rod, eliminating the cylinder from the pendulum. The rod length in this case was chosen to be $L=L_{r}+R=3.18 \mathrm{~cm}$. The moment coefficient behavior obtained varying the static angle of attack is shown in Fig. 7: considering its slope, a galloping instability for a simple rod in a soap film cannot be found. To verify this, we have performed experiments with rigid rods, varying their length and mass: none of the cases considered showed sustained oscillations. The observed instability for the rigid pendulum thus depends on its detailed geometrical shape.

\section{Control of the instability}

The galloping instability observed here may actually be useful if ways to harvest the energy of the oscillations are implemented. From another perspective, and considering that galloping may damage a structure, devising ways to passively control such instability is desirable. In this respect, we can take advantage of the fact the emergence of galloping strongly depends on the pendulum's geometry, which affects the production of lift forces induced by vortices's arrangement.

Thus, a natural idea to control such an instability is to modify the previous geometry. Following this line, we have examined the case of a pendulum with a flexible filament attached to the rear of the cylinder. The coupling between the rigid pendulum, the flexible filament and fluid vibrations is now non trivial and cannot be described anymore by a simple oscillator as in the previous section. Fig. 8 shows the bifurcation diagram we obtain when we add the filament to the rear of a pendulum that was found to exhibit large amplitude limit cycle oscillations without this extension, and we use the filament length as a bifurcation parameter

For short filaments, the system is found to maintain its self excited oscillatory dynamics, and both the pendulum and filament exhibit sustained, synchronized oscillations, whose frequency remains close to the one of a compound pendulum. However, when the filament length exceeds a critical value, it acts as a passive control device on the pendulum. damping out the oscillations of the rigid system. The filament's critical length is found to be about two disk diameters.

As shown in Fig. 9, for short lengths the filament oscillates in phase with the disk and the oscillations of the latter are sustained. In this situation, the pendulum and filament system behaves as a rigid object. For longer filaments, no instabilities are observed for the pendulum, whereas the filament flaps. When the pendulum is perturbed from the vertical position, the oscillations of the filament are out of phase with the oscillations of the pendulum. The motion of the disk is slowly damped out, but the flexible filament continues to flap. During this stage, a new frequency appears in the spectrum of the filament's 


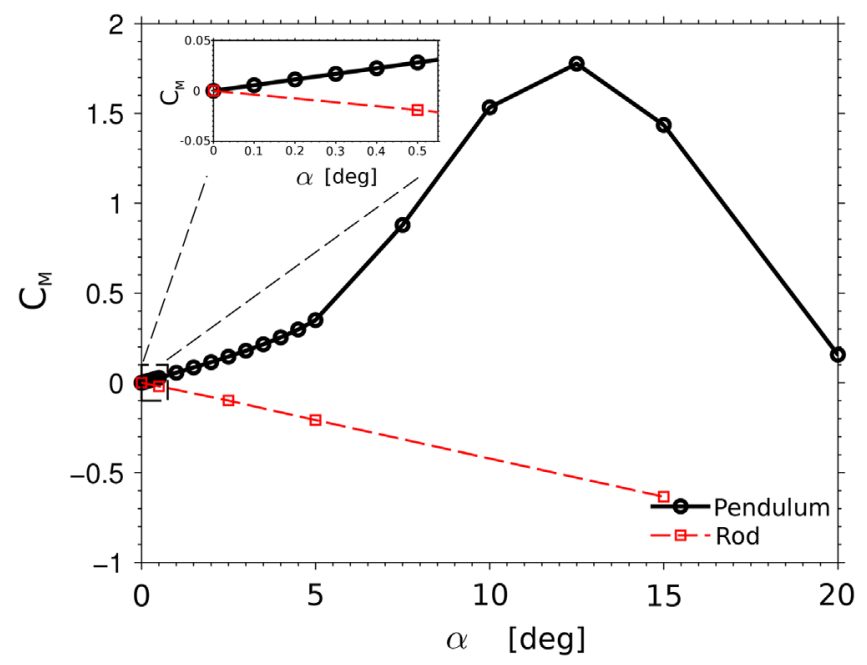

Fig. 7. Static momentum coefficient $C_{M}$ versus angle of attack for a pendulum and a straight rod. The inset shows the pendulum's coefficient slope at small angles of attack.

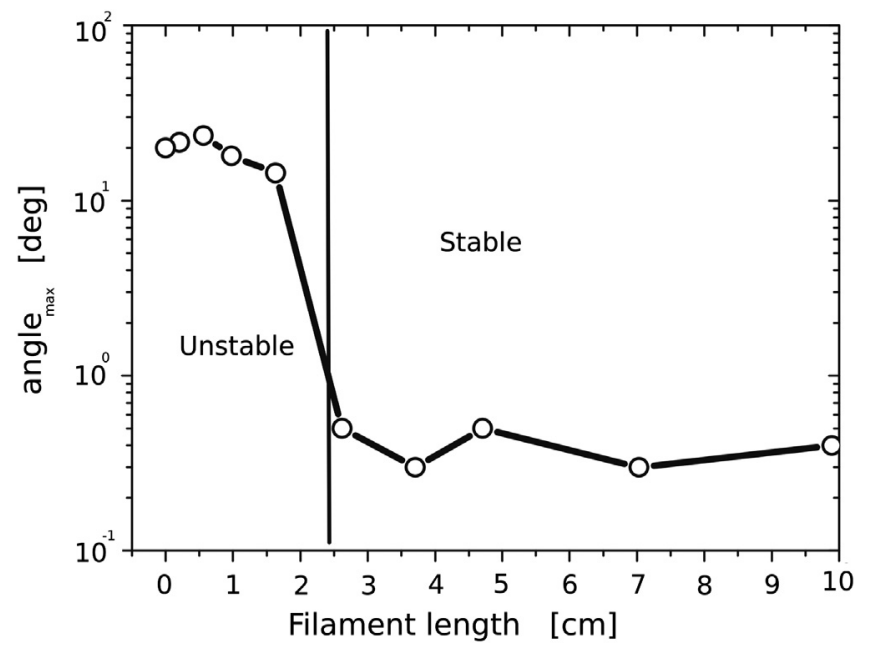

Fig. 8. Bifurcation diagram of pendulum's stability when a flexible filament is attached at its rear. Filament's length is used as a bifurcation parameter to control pendulum's oscillation. A bifurcation is found at a filament length of approximately $10 \mathrm{~mm}$. Parameters are fixed to be $U 180 \mathrm{~cm} / \mathrm{s}, R \quad 0.5 \mathrm{~cm}$, $L \quad 3 \mathrm{~cm}$ and $M \quad 0.1 \mathrm{~g}$. The filament diameter is about $200 \mu \mathrm{m}$.

tip evolution (see Fig. 9, right panel): the new frequency is higher than the pendulum's natural frequency, and can be associated to the natural elastic frequency of the filament triggered by the portion of it placed outside the cylinder wake.

A proper description of this control mechanism seems non trivial. An example highlighting the non trivial role of a passive appendage on the behavior of solid objects is discussed by Lacis et al. (2014), where it is shown how the appendage interacts with the wake, thus changing the flow structure and, in turn, the system's dynamics. Using simple concepts, one could argue that adding a filament to the rear of the pendulum increases added mass effects, which, according to Eq. (6), affects the stability of the system. Also, the presence of the filament induces non trivial changes in the rear flow dynamics, especially because the flow is $2 \mathrm{~d}$; topological flow variations in turn affect the fluid induced momentum on the body and could vary the sign of the moment coefficient derivative as per Eq. (6). However, we recall that Eq. (6) was derived assuming that the structure is a rigid body, whereas the filament is elastic. Because it has infinite degrees of freedom, it cannot be described by a simple oscillator, so these arguments become weak (they could hold for very short filaments).

These observations suggest that a passive elastic appendage introduces additional degrees of freedom and additional dynamics, which can stabilize self excited transverse oscillations of a rigid body embedded in a uniform stream, and therefore suppress the galloping instability. Note that although the filament is very light, it can stabilize structures that are orders of magnitude heavier. This might be thought of as a way to control galloping, which plagues a number of structures such as bridges, tall buildings, lighting poles and power cables. Also, since several natural organisms use passive appendages or flagella to swim, such passive instability control may also bring new information on the role of flexible appendages, and may be of interest in the analysis of locomotion strategies. 

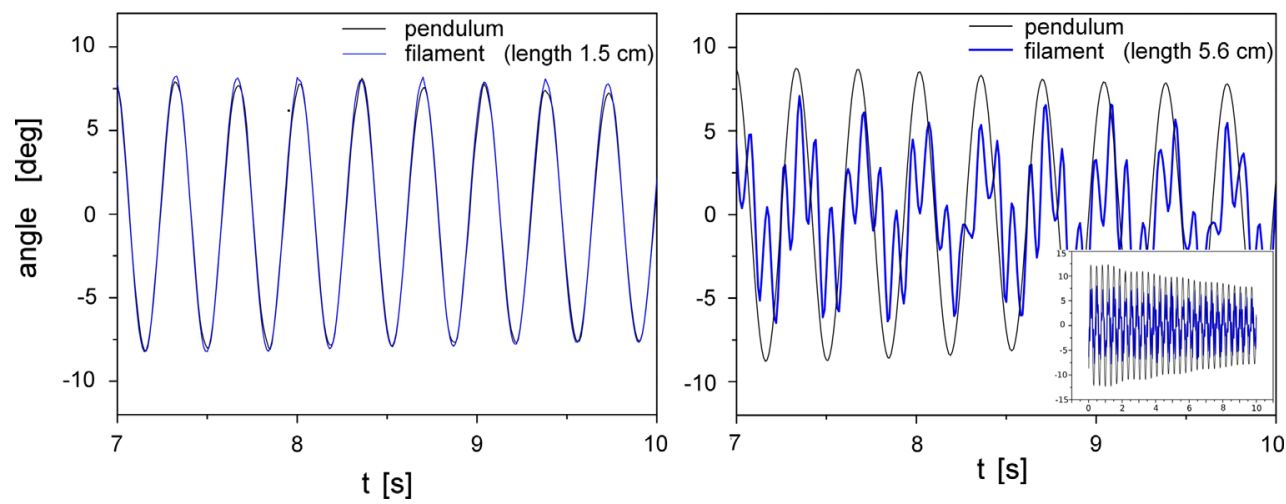

Fig. 9. Temporal evolution of the angle formed by the vertical axis with respect to disk's ( $R \quad 0.8 \mathrm{~cm})$ center of mass position (black) and filament's tip (blue). Two different filament lengths are considered: one below (left) and one above (right) the critical value. In the latter case the filament flaps with a frequency different from the pendulum's natural one, and damps the pendulum motion. Note that the behavior on the right is transient: for long times only the filament oscillations persist as illustrated by the inset. (For interpretation of the references to color in this figure caption, the reader is referred to the web version of this paper.)

\section{Conclusions}

Experiments have shown that a rigid pendulum in a two dimensional flow field and in a regime dominated by gravity exhibits self excited, sustained oscillations under a specific set of conditions. This instability is consistent with a galloping mechanism, and numerical simulations have been performed to confirm the nature of the instability. Further, we observe that adding a flexible filament at the rear of the pendulum stabilizes the rigid system, and may therefore be used to control galloping, or for devising locomotion strategies.

\section{Acknowledgments}

A.M. thanks the financial support for the computational infrastructure from the RITMARE project and the financial support from the PRIN 2012 project no. 2012MM3R79. Computational resources available within the INFN CINECA agreement are also acknowledged. H.K. thanks the IUF for financial support.

\section{References}

Allen, J.J., Smits, A.J., 2001. Energy harvesting eel. Journal of Fluids and Structures 15, 629-640, http://dx.doi.org/10.1006/jfls.2000.0355.

Amarouchene, Y., Kellay, H., 2004. Batchelor scaling in fast-flowing soap films. Physical Review Letters 93 (21), 214504, http://dx.doi.org/10.1103/ PhysRevLett.93.214504.

Amarouchene, Y., Kellay, H., 2005. Conformation statistics of a deformable material line in two-dimensional turbulence. Physical Review Letters 95 (July (5)), 054501, http://dx.doi.org/10.1103/PhysRevLett.95.054501.

Argentina, M., Mahadevan, L., 2004. Fluid-flow-induced flutter of a flag. Proceedings of the National Academy of Sciences 102 (6), 1829-1834, http://dx.doi. org/10.1073/pnas.0408383102.

Bagheri, S., Mazzino, A., Bottaro, A., 2012. Spontaneous symmetry breaking of a hinged flapping filament generates lift. Physical Review Letters 109 (October (15)), 154502, http://dx.doi.org/10.1103/PhysRevLett.109.154502.

Bandi, M.M., Concha, A., Wood, R., Mahadevan, L., 2013. A pendulum in a flowing soap film. Physics of Fluids 25 (4), 041702, http://dx.doi.org/10.1063/ 1.4800057.

Beal, D.N., Hover, F.S., Triantafyllou, M.S., Liao, J.C., Lauder, G.V., 2006. Passive propulsion in vortex wakes. Journal of Fluid Mechanics 549 (February), 385 , http://dx.doi.org/10.1017/S0022112005007925.

Blevins, R.D., 2001. Flow Induced Vibrations, 2nd Edition, Krieger Publishing Company, Malabar, Florida.

Boragno, C., Festa, R., Mazzino, A., 2012. Elastically bounded flapping wing for energy harvesting. Applied Physics Letters 100 (25), 253906, http://dx.doi. org/10.1063/1.4729936.

Chesshire, G., Henshaw, W.D., 1990. Composite overlapping meshes for the solution of Partial Differential Equations. Journal of Computational Physics 90 1-64, http://dx.doi.org/10.1016/0021-9991(90)90196-8.

Chomaz, J., 2001. The dynamics of a viscous soap film with soluble surfactant. Journal of Fluid Mechanics 442, 387-409, http://dx.doi.org/10.1017 S0022112001005213.

Henshaw, W.D., 1994. A forth-order accurate method for the incompressible Navier-Stokes equations on overlapping grids. Journal of Computational Physics 113, 13-25, http://dx.doi.org/10.1006/jcph.1994.1114.

Henshaw, W.D., 1998. Ogen: An Overlapping Grid Generator for Overture. Research Report UCRL-MA-132237. Lawrence Livermore National Laboratory. URL 〈http://overtureframework.org/documentation/ogen.pdf).

Jia, L.B., Yin, X.Z., 2008. Passive oscillations of two tandem flexible filaments in a flowing soap film. Physical Review Letters 100 (June (22)), 228104, http: //dx.doi.org/10.1103/PhysRevLett.100.228104.

Kellay, H., Goldburg, W.I., 2002. Two-dimensional turbulence: a review of some recent experiments. Reports on Progress in Physics 65, 845-894, http://dx doi.org/10.1088/0034-4885/65/5/204.

Kellay, H., Wu, X., Goldburg, W., 1998. Vorticity measurements in turbulent soap films. Physical Review Letters 80 (January (2)), $277-280$.

Kellay, H., Wu, X.L., Goldburg, W.I., 1995. Experiments with turbulent soap films. Physical Review Letters 74 (20), 3975-3978, http://dx.doi.org/10.1103/ PhysRevLett.74.3975. 
Lacis, U., Brosse, N., Ingremeau, F., Mazzino, A., Lundell, F., Kellay, H., Bagheri, S., 2014. Passive appendages generate drift through symmetry breaking. Nature Communications 5, 5310, http://dx.doi.org/10.1038/ncomms6310.

Lauga, E., Powers, T.R., 2009. The hydrodynamics of swimming microorganisms. Reports on Progress in Physics 72 (September (9)), 096601, http://dx.doi. org/10.1088/0034-4885/72/9/096601.

Lemaitre, C., Hémon, P., de Langre, E., 2005. Instability of a long ribbon hanging in axial air flow. Journal of Fluids and Structures 20 (October), 913-925, http://dx.doi.org/10.1016/j.jfluidstructs.2005.04.009.

Liao, J.C., Beal, D.N., Lauder, G.V., Triantafyllou, M.S., 2003. Fish exploiting vortices decrease muscle activity. Science 302 (November), 1566-1569, http://dx. doi.org/10.1126/science.1088295.

Obligado, M., Puy, M., Bourgoin, M., 2013. Bi-stability of a pendular disk in laminar and turbulent flows. Journal of Fluid Mechanics 728 (July), 2, http://dx. doi.org/10.1017/jfm.2013.312.

Orchini, A., Mazzino, A., Guerrero, J., Festa, R., Boragno, C., 2013. Flapping states of an elastically anchored plate in a uniform flow with applications to energy harvesting by fluid-structure interaction. Physics of Fluids 25 (9), 097105, http://dx.doi.org/10.1063/1.4821808.

Provansal, M., Leweke, T., Schouveiler, L., Aprile, C., 2005. Oscillations and vortex-induced vibrations of a tethered sphere in a flow. Journal of Flow Visualization and Image Processing 12 (1), 29-44, http://dx.doi.org/10.1615/JFlowVisImageProc.v12.i1.40.

Rutgers, M.A., Wu, X.L., Daniel, W.B., 2001. Conducting fluid dynamics experiments with vertically falling soap films. Review of Scientific Instruments 72 (7), 3025, http://dx.doi.org/10.1063/1.1379956.

Shelley, M., Vandenberghe, N., Zhang, J., 2005. Heavy flags undergo spontaneous oscillations in flowing water. Physical Review Letters 94 (March (9)), 094302, http://dx.doi.org/10.1103/PhysRevLett.94.094302.

Spalart, P.R., 1998. Airplane trailing vortices. Annual Review of Fluid Mechanics 30 (January), 107-138, http://dx.doi.org/10.1146/annurev.fluid.30.1.107.

Williamson, C.H.K., Govardhan, R., 2004. Vortex-induced vibrations. Annual Review of Fluid Mechanics 36 (January), 413-455, http://dx.doi.org/10.1146/ annurev.fluid.36.050802.122128.

Zhang, J., Childress, S., Libchaber, A., Shelley, M., 2000. Flexible filaments in a flowing soap film as a model for one-dimensional flags in a two-dimensional wind. Nature 408, 835-839, http://dx.doi.org/10.1038/35048530.

Zhu, L., Peskin, C.S., 2002. Simulation of a flapping flexible filament in a flowing soap film by the Immersed Boundary Method. Journal of Computational Physics 179 (July (2)), 452-468, http://dx.doi.org/10.1006/jcph.2002.7066.

Zhu, L., Peskin, C.S., 2003. Interaction of two flapping filaments in a flowing soap film. Physics of Fluids 15 (7), 1954 , http://dx.doi.org/10.1063/1.1582476. 